Musées, Patrimoine et Culture scientifiques et techniques

$111 \mid 2007$

mai - juin 2007

\title{
Le droit d'entrée dans la perspective du don
}

\section{François Mairesse}

URL : http://journals.openedition.org/ocim/761

DOI : 10.4000/ocim.761

ISSN : 2108-646X

Éditeur

OCIM

Édition imprimée

Date de publication : 1 mai 2007

Pagination : 23-30

ISSN : 0994-1908

Référence électronique

François Mairesse, «Le droit d'entrée dans la perspective du don », La Lettre de I'OC/M [En ligne],

111 | 2007, mis en ligne le 15 mars 2011, consulté le 19 avril 2019. URL : http://

journals.openedition.org/ocim/761; DOI : 10.4000/ocim.761 


\section{Le droit d'entrée \\ dans la perspective du don}

François Mairesse *

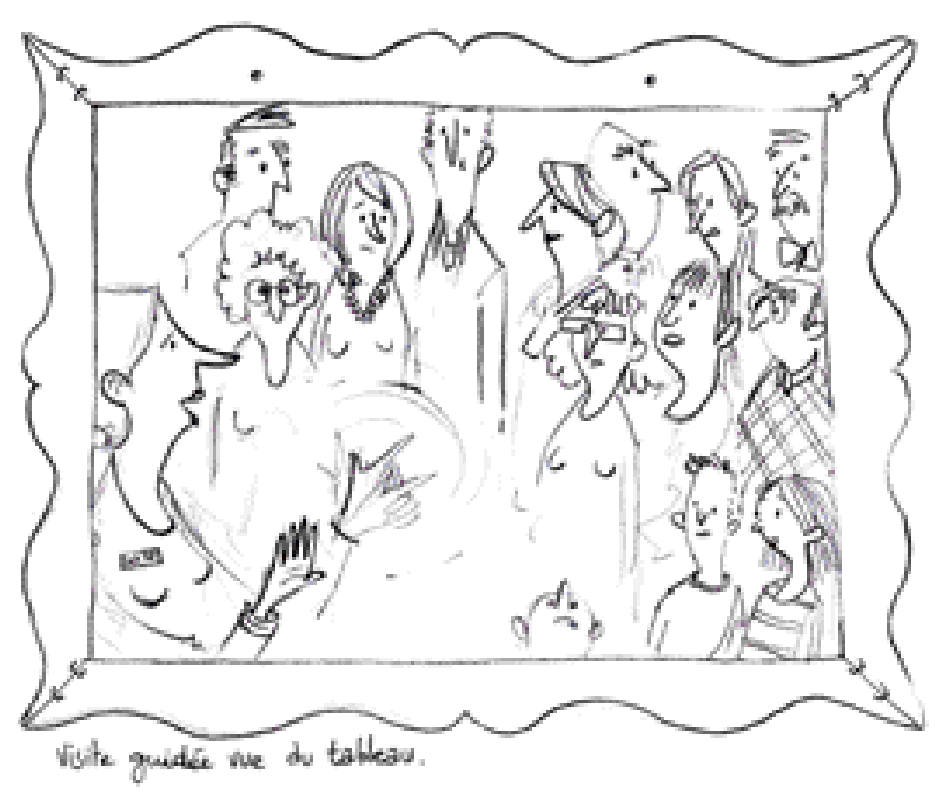

두 Coline Desclides

* François Mairesse est directeur du musée Royal de Mariemont mairesse@musee-mariemont.be
Face à la querelle qui oppose les tenants d'un droit d'entrée au musée et les partisans de la gratuité, l'auteur présente une troisième voie basée sur le don, un autre mécanisme d'échange du paysage muséal. Cette réflexion sur la question du don à l'entrée du musée, qui repose sur une participation active du visiteur, apporte également un éclairage différent sur les débats concernant l'application des règles du marché à la gestion des musées.

Le landerneau muséal a été récemment animé par une certaine agitation autour de la question du droit d'entrée. D'un côté, quelques musées provoquaient l'irritation, les modifications tarifaires qu'ils avaient entreprises étant jugées trop commerciales ; de l'autre, plusieurs instances publiques décidaient de rendre gratuite l'entrée aux musées dont elles avaient la charge. On sait, bien sûr, que les activités des musées sont relativement coûteuses; la question qui nous préoccupe ici consiste à savoir si une partie du coût de leur fonctionnement pourrait être directement prise en charge par le consommateur ou s'il revient à l'État de se charger, seul, de cette tâche, de manière à assurer un accès libre pour tous. D'une part, donc, la mise en marché dont il est souvent question au sein des musées ne répond qu'à une portion réduite de leurs besoins en fonctionnement ; d'autre part, le débat est déjà ancien : si le développement des techniques de marketing au sein des musées a incontestablement influencé la discussion, les premières demandes pour l'instauration d'un 
droit d'entrée remontent, en France, à la seconde moitié du XIX ${ }^{\mathrm{e}}$ siècle, alors que déjà le manque criant de ressources affectées aux musées par les pouvoirs publics amène conservateurs et amateurs à espérer d'autres moyens pour ces établissements (1). Les arguments invoqués par les partisans de l'une ou l'autre solution ont atteint, avec le temps, un haut niveau de sophistication. Désormais, c'est à partir d'enquêtes statistiques et d'analyses économiques que l'un et l'autre points de vue sont évoqués et, à ce niveau de rationalité économique, ce sont sans doute les arguments en faveur de la mise en marché qui semblent les plus percutants.

A priori, tout oppose détracteurs et partisans du droit d'entrée : d'un côté, les premiers invoquent la liberté d'accès à toutes les classes sociales et les nouvelles modalités de visite que permet l'entrée libre, alors qu'un prix d'entrée, même modique, induit une barrière sinon véritablement économique, du moins psychologique ; de l'autre, les seconds dénoncent les carences du financement public et soulignent les nouvelles méthodes permettant de cibler les publics les plus fragilisés (faire entrer gratuitement ceux qui ne peuvent vraiment rien payer) et amener toute personne qui en a les moyens à régler son entrée - notamment les touristes qui, depuis le XIX siècle, sont régulièrement cités comme une source de revenus considérable qu'il serait aberrant de négliger. De manière générale, la querelle porte en fait sur le rôle de l'État à assumer un soutien financier suffisant ou, à l'inverse, sur la capacité au marché à réguler de manière optimale la rencontre entre la demande des consommateurs et l'offre muséale, les partisans du droit d'entrée soulignant tous que si des moyens supplémentaires leur étaient fournis, ils seraient bien évidemment favorables à l'entrée gratuite. Aux arguments quantifiés apportés par les uns répondent les arguments de droit à l'éducation ou de droit à la culture des autres.

Ces deux points de vue, apparemment inconciliables, présentent cependant un trait commun que l'on pourrait définir comme une conception relativement restreinte de la participation du visiteur au sein du débat. Somme toute, il revient à ce dernier d'accepter ou non les termes d'un échange qui lui est imposé et dont le contrat repose sur des règles claires mais peu modulables : payer et obtenir le droit de visiter le musée ou refuser et rester à l'extérieur. En tout état de cause, il n'y a pas de discussion sur le prix - mis à part quelques cas exceptionnels engendrant le remboursement de la prestation, s'apparentant à une sorte de garantie "satisfait ou remboursé » en vigueur plus ou moins tacitement au sein du marché.

\section{Les musées et le don}

Cette querelle, largement relayée par la presse, éclipse presque totalement un autre mécanisme d'échange pourtant omniprésent au sein de l'univers muséal et que l'on pourrait identifier comme une forme de don. L'échange par don est en effet probablement, autant que les échanges réglés au sein de l'économie de marché et ceux gérés selon les modalités établies par les pouvoirs publics, non seulement indissociable du fonctionnement traditionnel du musée, mais sans doute une composante indispensable de son développement.

On sait l'ancienneté des échanges par dons, en ce compris au sein de nos propres sociétés. Pratique omniprésente au sein des cités grecques et romaines - l'historien Paul Veyne a analysé cette pratique au sein de la sphère publique (l'évergétisme) - le don permet à l'Église, à partir du Moyen-Âge et jusqu'à la fin de

\section{François}
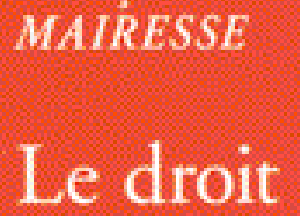
d'entrer au musée l'Ancien Régime, le développement qu'on lui connaît. L'institution du don, qu'elle se produise du vivant du donateur ou de manière posthume, par legs, est intimement mêlée à la création des premiers musées, qu'il s'agisse des collections Grimani données à la ville de Venise (1523 et 1587), du cabinet Ashmole/Tradescant légué à Oxford (1677) ou des collections Boisot léguées à Besançon (1694). À partir de la fin du XIX ${ }^{\mathrm{e}}$ siècle, de plus en plus de musées sont créés à partir de collections privées offertes à la collectivité ; qu'il s'agisse du château de Chantilly (le duc d'Aumale) ou des musées Jacquemart-André, Nissim de Camondo et CognacJay, pour n'en citer que quelques 
exemples à Paris et ses environs. C'est sans doute aux États-Unis que le financement et l'enrichissement des musées par des dons connait le plus d'ampleur, le fonctionnement même de ces établissements reposant en grande partie sur le principe des fondations privées, œuvres de milliardaires mécènes qui n'hésitent pas à doter richement le capital de ces institutions.

Le recours aux dons est, d'une manière ou d'une autre, influencé par les autres modes d'échanges. D'une certaine manière, l'État, à partir du XIX siècle, prend en charge nombre de domaines autrefois dévolus à la sphère privée et administrés par des sociétés charitables. Après la Seconde Guerre mondiale, la politique économique est largement influencée par le recours à l'État providence, peutêtre au détriment d'un certain esprit philanthropique dans nos régions. À partir du dernier quart du $\mathrm{XX}^{\mathrm{e}}$ siècle, la part de l'État diminue quelque peu au profit du marché, mouvement qui s'amplifie encore avec l'effondrement progressif du bloc de l'Est. La pensée dite néo-libérale, largement influencée par les pays anglo-saxons, ne manque pas de produire ses effets sur le financement des musées, dès lors souvent moins soutenus par l'État. Il est vrai que le rôle moins important des pouvoirs publics, en Grande-Bretagne et aux États-Unis, apparaît comme favorable au mécénat et à la philanthropie, engendrant généralement une attitude de plus grande responsabilité des élites économiques en faveur du développement de la culture et, notamment, le soutien du monde des musées.
Le don, au niveau des musées, s'exprime de diverses manières que l'on pourrait réunir en trois groupes principaux, selon qu'ils privilégient l'argent, les objets de collections ou le temps à leur consacrer. Les dons d'argent sont souvent générés, dans nos régions, par le truchement d'associations d'amis qui collectent des fonds au bénéfice du musée qu'elles représentent, souvent afin d'enrichir les collections. Parfois, l'appel aux dons - les levées de fonds ou fundraising - est organisé par les musées eux-mêmes, surtout lorsque ceux-ci disposent d'une certaine autonomie financière. Le don de spécimens ou d'objets de collections constitue, surtout pour les musées d'art, une spécificité propre au système patrimonial qui permet de distinguer ces derniers (avec les bibliothèques et les centres d'archives), de manière générale, des autres institutions à but non lucratif. Le visage de la plupart des musées ne serait pas celui que l'on connaît sans l'intervention de ces donateurs, offrant le fruit de leur passion afin de lui garantir, notamment, une certaine pérennité. Enfin, le bénévolat constitue un troisième mode de don, bien sûr différent des deux premiers, mais en dehors lui aussi, comme ces derniers, de l'échange économique classique. Sans doute le bénévolat constituet-il une activité s'épanouissant plus souvent sur le continent américain qu'en Europe : aux États-Unis, un habitant de plus de dix-huit ans sur 480 travaillerait bénévolement dans les musées (2)! Dans nos régions, de très nombreuses petites institutions muséales, fonctionnant selon le régime des associations, fonctionnent essentiellement sur base de prestations de ce type.

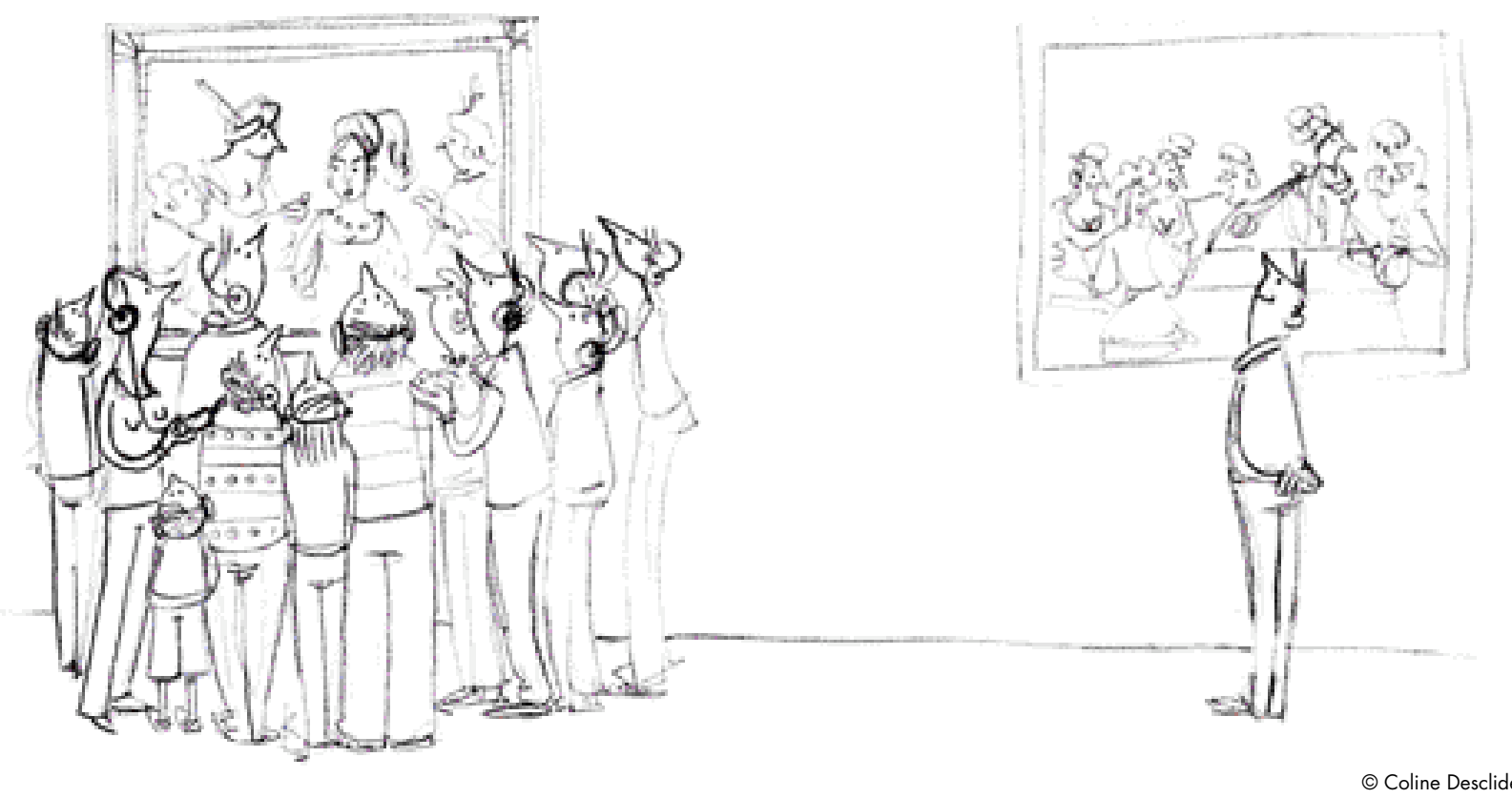


Marcel Mauss, parmi les premiers, a tenté de définir la mécanique interne à ces élans de générosité apparemment volontaires, en réalité parfois obligatoirement prestés et, en tout cas, savamment codifiés. L'essai sur le don, publié en 1924 sur la base d'une abondante documentation rassemblant des informations provenant des quatre coins du globe, conclut à la persistance d'une logique se déclinant en trois temps (par ailleurs déjà énoncée par Sénèque) : donner, recevoir et rendre ${ }^{(3)}$. Sans doute ce type de mécanisme s'observe-t-il plus visiblement au sein de sociétés traditionnelles (les Tobriandais ou les Kwakiutl, que Mauss cite abondamment) que dans nos sociétés actuelles. Sans doute aussi le don prendil une place d'autant plus significative au sein d'une société ou d'un groupe social déterminé lorsque les membres qui la composent connaissent les règles implicites du jeu en vigueur. Un jeu dont les aspects généreux, aux premiers abords, semblent si séduisants, mais dont les caractéristiques négatives (écraser quelqu'un par un don qu'il ne pourra rendre ou l'obliger à la reconnaissance éternelle) sont soulignées par Mauss. De tels principes ne sont d'ailleurs pas étrangers au monde des musées : les conditions évoquées par certains mécènes, les exigences de certains donateurs peuvent s'avérer d'une grande difficulté pour le fonctionnement d'un musée, a fortiori lorsque le don est tellement important qu'il ne peut être réellement refusé et qu'il oblige cependant le musée à la reconnaissance inconditionnelle.

Tour à tour, la mécanique publique et celle du marché semblent vouloir s'imposer au détriment du don.

Si le développement de la puissance publique amène progressivement la disparition d'un grand nombre d'institutions charitables et philanthropiques (notamment afin de contrer les aspects négatifs engendrés par ces dernières), la logique hégémonique de l'économie de marché, à la fin du XX $X^{\mathrm{e}}$ siècle, tente d'expliquer par le fonctionnement de l'homo œeconomicus tous les mécanismes d'échange, en ce compris ceux régissant traditionnellement la sphère familiale et ceux que l'on regroupait sous l'étiquette de mécénat, de philanthropie ou de don. Un certain nombre d'auteurs (notamment Alain
Caillé ou Jacques Godbout, rassemblés autour de l'œuvre du sociologue français, au sein de la Revue $d u$ M.A.U.S.S.) opposés à cette seule alternative État/Marché, revendiquent cette troisième voie du don, fondée sur un paradigme anti-utilitariste expliquant autrement les termes de l'échange non-marchand. Cette dernière perspective, si elle est loin d'être aussi solidement acceptée que les hypothèses sur lesquelles reposent l'économie de marché, permet tout au moins d'isoler un certain nombre de facteurs existant au sein du musée et qui ne sont pas pris en compte lors des débats sur le droit d'entrée.

\section{Le don à l'entrée}

Un certain nombre de contributions plus ou moins volontaires peuvent être en effet demandées à l'entrée du musée - voire durant la visite, " n'oubliez pas le guide »- dont la logique rompt avec celle du droit d'entrée classique.

Dans la plupart des musées gratuits, par exemple en Grande Bretagne au British Museum et à la National Gallery qui ont continué, contre vents et marées, à poursuivre une politique de gratuité, un tronc accueillant les donations est présenté à l'entrée de l'établissement, dont le produit est loin d'être négligeable. D'autres musées, comme le Metropolitan Museum de New York, ont instauré depuis de nombreuses années un système fondé sur une contribution volontaire mais obligatoire : le visiteur est libre de donner ce qu'il souhaite, mais il doit passer par la billetterie. Les nécessités budgétaires de même que 
le renforcement des techniques mercatiques ont amené la mise en place d'un conditionnement social de plus en plus important : le montant suggéré de la donation est fort élevé, la donation une fois remise s'affiche, en grand, sur le compteur de la caisse enregistreuse, afin que le public dans la file opère un contrôle subtil, au point que nombre de visiteurs conditionnés par la fréquentation des autres musées pensent que le Metropolitan est tout simplement devenu payant. Cette pratique qui permet à n'importe quel amateur régulier d'entrer pour 10 cents dans l'un des plus grands musées du monde rapporterait cependant en moyenne autant que le système de billetterie mis en place au musée du Louvre ${ }^{(4)}$. Un système plus ou moins identique existe au musée des Beaux-Arts de Montréal, tandis que d'autres expériences, plus ou moins fructueuses, ont été tentées depuis une trentaine d'années de par le monde, notamment à Londres (Victoria \& Albert Museum), à Boston ou à Morlanwelz (musée royal de Mariemont, Belgique).

Si un certain nombre de détracteurs s'opposent à de telles propositions, parfois comparées à un pourboire ou à une aumône, force est de reconnaître que le principe d'un tel mécanisme est singulièrement différent des autres, puisque le prix est laissé à l'appréciation du consommateur qui joue donc un rôle nettement plus actif. Plus que la gratification donnée après une prestation spécifique (pourboire), ce procédé s'apparente aux modes de perception en vigueur dans les églises, ashrams, mosquées et autres lieux liés le plus souvent à la spiritualité. L'entrée dans la plupart des églises, en Europe, est la plupart du temps libre, mais des troncs incitent le fidèle à contribuer à l'entretien de l'édifice ou à un certain nombre d'œuvres spécifiques. À l'inverse, il arrive actuellement que certaines des églises dont le caractère patrimonial est particulièrement marqué (notamment par la présence d'œuvres d'art importantes) agissent de plus en plus comme des entreprises commerciales, tentant de vendre cartes et souvenirs, d'initier - en Italie surtout - un système d'éclairage payant, voire d'imposer un droit d'entrée, au risque de choquer les fidèles. La logique de la collecte durant les offices constitue également un mode de perception utilisé dans de tels lieux, bien quill soit nettement moins fréquent pour les musées, sauf lors de certains galas de bienfaisance. Nombre d'organisations caritatives (équipes de médecins, recherche contre certaines maladies) ont développé ces pratiques de manière très sophistiquée, utilisant notamment régulièrement la force de persuasion des médias.

\section{Pourquoi donne-t-on ?}

S'il est certes peu utilisé, le don à l'entrée présente une alternative qui permet de s'interroger sur les raisons de cet échange particulier. Les raisons d'un échange commercial classique sont en effet assez claires : on paye pour consommer, pour visiter en l'occurrence. Mais pourquoi donner ? La réponse est évidemment nettement plus complexe, puisque les règles de l'échange ne sont pas écrites. De manière générale, sans doute, c'est la contrainte sociale qui permet d'expliquer partiellement les raisons du don (notamment lors d'une collecte ou d'un don obligatoire) : faire comme les autres ou comme dans d'autres lieux (où l'on paye), de crainte de subir la désapprobation tacite du groupe. Mais pour un certain nombre de visiteurs, les raisons peuvent s'avérer différentes, se déclinant en fonction de la personnalité, de l'éducation ou des histoires de vie : on peut donner de manière compulsive, pour améliorer son statut social, par tradition familiale... Le lien commun entre toutes ces raisons tient, semble-t-il, à la relation au groupe ou à « l'autre », relation qui n'existe pas ou du moins que l'on s'efforce de limiter lors d'une transaction commerciale ou administrative. Je n'ai aucune stratégie spécifique lorsque je commande une place de cinéma ou que je renouvelle ma carte d'identité à la mairie ; la chose est parfois différente lorsque je participe à une quête ou que je donne à

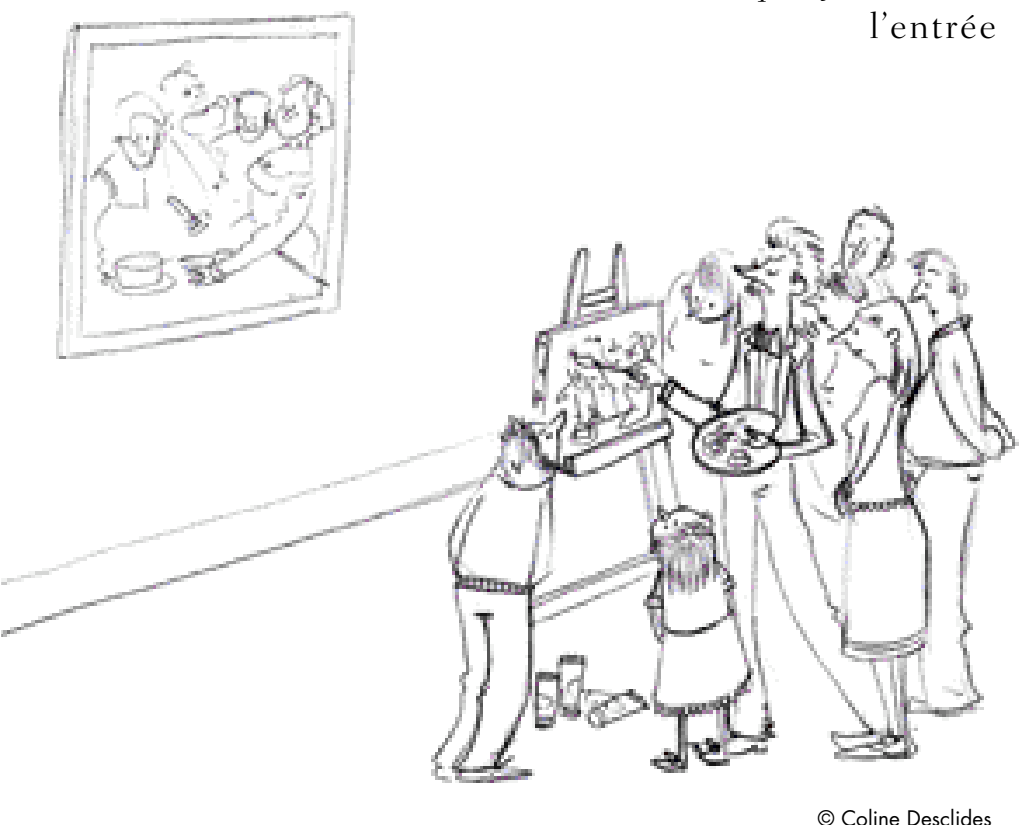


d'un sanctuaire. Elle l'est encore plus lorsque je connais la personne à qui je donne (ce qui est plutôt rare, à l'entrée d'un musée, mais s'avère nettement plus courant si j'offre un tableau à un conservateur pour son établissement). Ce jeu de relations humaines existe donc à deux niveaux, entre moi, les autres visiteurs et le personnel du musée. À celui-ci s'ajoute une dimension diachronique, elle aussi différente du cadre strict de l'échange commercial ou administratif : je ne suis pas tenu de saluer mon percepteur des impôts ou mon garagiste dans la rue (à moins que se soient tissés des relations amicales qui dépassent justement l'échange), tandis que je me dois absolument de saluer un donateur si je suis conservateur. Par delà l'échange, une relation s'est souvent tissée, celle justement qui explique la dette et le retour du don (par quelque forme que ce soit). C'est aussi cette dynamique, dont il est parfois difficile de bien connaître l'origine, fondée sur l'histoire des relations humaines voire spirituelles, qui peut opérer sur un don à l'entrée : je donne en souvenir d'une agréable visite, d'un conservateur attentif ou d'un service rendu, parfois même ce geste constitue-t-il la résultante d'une aventure passée dans un autre musée ou en souvenir d'une personne chère ayant un rapport avec les musées : le retour d'un don ne passe pas toujours par celui qui l'a envoyé.

La question peut être également renversée : que donne le musée, qui amène en retour cet échange particulier ? Question complexe, à laquelle une seule réponse ne saurait suffire. Qu'il soit temple ou école (selon la définition de Benjamin Gilman, départageant ainsi, dans leur essence, musée d'art et musée de science $\left.{ }^{(5)}\right)$, c'est peut-être plus parce qu'il est lieu de transformation de l'être qu'il appartient à la catégorie des institutions fonctionnant aisément selon les principes du don. Au même titre que la bibliothèque, l'école, l'église ou l'hôpital, l'homme peut espérer, en entrant dans le musée, une certaine transformation. Pas une transformation physique comme à l'hôpital, bien sûr, mais une certaine initiation, tantôt historique ou scientifique, tantôt esthétique, offrant à chacun une leçon de civilisation, des clés vers les plus hauts sommets de la réalisation humaine. Cette attitude de révérence par rapport aux hauts projets culturels du musée, que l'on perçoit inscrite dans la pierre des musées construits au $\mathrm{XIX}^{\mathrm{e}}$ siècle, se retrouve encore parfois, de manière certes un peu altérée, au sein des grandes institutions visitées, à la manière des cathédrales, par les pèlerins d'un nouveau genre en quête des nouvelles œuvres sacrées que constituent la Joconde, la momie de Ramsès II ou la capsule d'Apollo 11.
Bien sûr, par rapport aux systèmes classiques de tarification du musée, le don semble nettement plus aléatoire puisqu'il repose sur une participation active du visiteur, ce qui amène nombre de responsables à relativiser son efficacité, doutant ainsi de la volonté de participation du public à un tel projet. Cette hypothétique fragilité constituait pourtant presque la norme pour la plupart des institutions spirituelles et nombre d'organisations non gouvernementales caritatives. Pourquoi certains musées ne parviendraient-ils pas à fonctionner de cette manière ?

Il convient, en outre de percevoir cette question à l'aune de l'ensemble des dons transitant autour du musée. C'est parfois pour que l'entrée demeure gratuite, libre pour tous, que certaines donations sont effectuées. Ainsi, au musée de Cincinnati, plusieurs dons très importants ont permis de garantir la pérennité de l'entrée libre au musée, d'abord à raison d'un jour par semaine (depuis 1906, suite à une dotation spéciale de Mary Emery), puis pour les enfants (dotation de la Cincinnati financial Corporation) et, récemment pour l'ensemble du public (dotation de la Richard and Lois Rosenthal Foundation). À l'inverse, un grand mécène britannique, Sir Denis Mahon, a annulé en 1997 un legs de plusieurs tableaux de maîtres qu'il s'apprêtait à faire à la Walker Art Gallery de Liverpool, qui venait d'instaurer un système d'entrée payante ${ }^{(6)}$.

Il serait illusoire de considérer la question du don comme une solution à tous les problèmes du fonctionnement des musées. Cette perspective permet cependant de porter un autre regard sur les débats concernant l'immixtion plus ou moins importante des règles du marché au sein des musées. En fonction de ce point de vue, il est difficile de prétendre qu'une relation strictement organisée selon les principes marchands ou ceux de la gestion publique offre une solution définitive à tous les problèmes liés à la venue des visiteurs au sein d'un musée. La question du don à l'entrée induit d'abord un autre type d'investissement, certes réduit par rapport à certaines actions possibles (bénévolat, don d'objets), mais dont la philosophie sous-jacente implique un positionnement différent du visiteur/citoyen par rapport au musée, puisqu'il lui revient de décider (plus ou moins) librement de l'un des termes de l'échange : sa participation. Cette espace de liberté, mais surtout d'incertitude pour le conservateur, n'est pas sans difficultés pour la gestion d'un musée. La dépendance de l'établissement par rapport au bon vouloir du visiteur est peut-être, dans cette optique, encore plus 


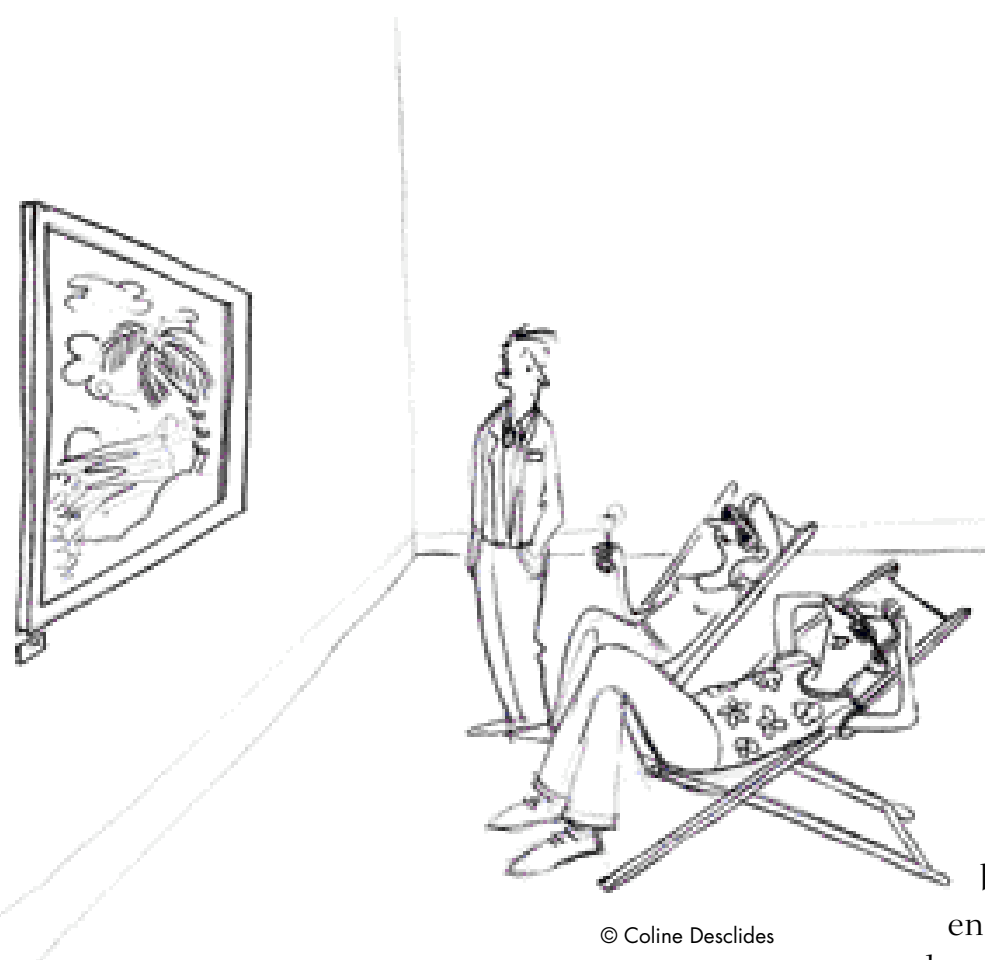

données pour financer les musées. Une telle question est, depuis le début des années 1990, au cœur de l'actualité, la pensée économique contemporaine s'interrogeant sur les nécessités de l'intervention publique. C'est pour cette raison que se sont développés nombre d'arguments permettant de justifier l'existence des musées. L’Audit Commission britannique avait répertorié au début des années 1990 ces raisons dans l'ordre suivant : le développement de la qualité de vie dans une région, le développement du tourisme, le développement économique, le support à la recherche et à l'éducation, la conservation du patrimoine ${ }^{(7)}$. On sait combien ces premières fonctions ont été mises en valeur ces dernières années, au point de devenir, dans l'esprit de certains décideurs, les

difficile à gérer pour le musée que celle, déjà délicate, qui se présente dans une relation de marché (visiteur/consommateur); elle induit surtout la nécessité d'une relation différente avec les utilisateurs de l'institution muséale, plus proche des modèles qui ont pu être élaborés par certains auteurs (John Cotton Dana, Patrick Geddes, John Kinard, pour ne citer que quelques pionniers) engagés dans la muséologie communautaire. Une relation exigeante et complexe, qui n'est pas sans risques autant pour le musée que pour le visiteur progressivement amené à devenir acteur au sein de l'établissement.

Sans doute les responsables de beaucoup de musées considéreront-ils avec un certain scepticisme une telle mécanique pour l'entrée de leur établissement, mais il est évident qu'au moins à un certain niveau celui du don de collections - un conservateur ne peut rester dans le seul statut d'agent des services publics et doit s'investir personnellement dans une relation qui n'a plus grand-chose à voir avec celle que l'on peut envisager selon l'application stricte des règles administratives ou de marché. On sait que de telles relations, dès lors qu'elles touchent deux personnes, ne sont pas exemptes de risques, tant pour le conservateur que le donateur.

Quoiqu'il en soit, la perspective du don offre un éclairage particulièrement intéressant sur les raisons raisons essentielles pour lesquelles il convient de créer, de développer ou d'entretenir des musées. Les projets initiés à Bilbao n'offrent-ils pas la preuve d'une réussite économique extraordinaire ? Dans une perspective régie strictement par les règles du marché, de telles missions peuvent logiquement se développer sur base d'un financement provenant partiellement des consommateurs eux-mêmes. De même, et toujours selon une perspective économique, l'intervention de l'État, lorsqu'il s'agit de développer le milieu de vie d'une région ou son potentiel touristique et économique, s'accommode aisément de la logique économique privilégiant la perception maximale d'un droit d'entrée en fonction d'études de marketing ciblées. En suivant jusqu'au bout cette logique, ne faudrait-il pas construire de nouvelles cathédrales comme celle de Chartres ? De tels arguments s'effondrent, en effet, face à la philosophie du don : si l'on peut concevoir que l'on donne pour affronter le temps (conservation) ou pour élever les esprits (éducation), il est rarissime que l'on donne pour le développement de l'économie et du tourisme de sa région.

La pensée à partir d'une philosophie du don permet ainsi de souligner les différences fondamentales existant entre certaines missions à partir desquelles s'articule l'activité des musées. D'une manière ou d'une autre, l'hégémonie de l'un de ces modes de fonctionnement que constituent le don, l'économie de marché ou la logique publique, se fait nécessairement au 
détriment de l'accomplissement de certaines missions que le musée pourrait remplir. Question de choix, le jeu reste ouvert.

Car pourquoi pas, après tout, un musée développé uniquement à des fins touristiques ? Au vu de la fréquentation de certains établissements, et non des moindres, ne s'agit-il pas là déjà d'un fait plus ou moins avéré ? Dès lors, pourquoi pas, effectivement, un accès strictement régenté par les règles du marché ? Et bien sûr, une sanction identique à celles du marché en cas d'insuccès : la faillite et la disparition. À moins, bien sûr, qu'une certaine sagesse permette de reconnaître que sous le vocable de musées, un grand nombre d'établissements fort peu similaires puissent voir le jour, remplissant des fonctions apparemment identiques mais pour des raisons parfois très dissemblables et recourant pour ce faire à des modes de financement très différents. Selon cette acception, le monde des musées ne serait-il pas ainsi plus proche de l'hétérogénéité qui prévaut au sein de notre humanité ?

\section{Notes}

(1) Mairesse, F. Le droit d'entrer au musée. Bruxelles : Labor, 2005.

(2) American Association of Museums, Museums Count. Washington : AAM, 1994, p. 81.

(3) Mauss, M. Essai sur le don, forme et raison de l'échange dans les sociétés archaïques, Sociologie et anthropologie, Paris : Presses universitaires de France, 1950

(4) Jerosme, N. Un coût élevé pour un effet incertain (entretien avec F. Cachin), Le Journal des Arts, 22 mai 1998, p. 8.

(5) Gilman, B.-I. Museums Ideals of Purpose and Methods. Cambridge: Harvard University Press, 1923.

(6) Pour le premier exemple, voir le site Internet du Cincinnati Art Museum (www.cincinnatiartmuseum.org), pour le second, voir Jerosme, N. C'est gratuit et ça peut rapporter gros, Le Journal des Arts, 22 mai 1998, p. 9.

(7) Audit Commission, The Road to Wigan Pier ? Managing Local Authority Museums and Art Galleries. London : HMSO, 1991. 